\title{
B-cell subset alterations and correlated factors in HIV-1 infection
}

\author{
Simone Pensieroso ${ }^{a}$, Laura Galli ${ }^{b}$, Silvia Nozzab ${ }^{b}$ Nicolas Ruffinc, \\ Antonella Castagna ${ }^{b}$, Giuseppe Tambussi ${ }^{b}$, Bo Hejdeman ${ }^{d}$, \\ Donatella Misciagna ${ }^{\mathrm{e}}$, Agostino Riva ${ }^{\mathrm{e}}$, Mauro Malnati ${ }^{\mathrm{f}}$, \\ Francesca Chiodi ${ }^{\mathrm{c}, *}$ and Gabriella Scarlatti ${ }^{\mathrm{a}, *}$
}

\begin{abstract}
Objectives: During HIV-1 infection, the development, phenotype, and functionality of $B$ cells are impaired. Transitional B cells and aberrant B-cell populations arise in blood, whereas a declined percentage of resting memory B cells is detected. Our study aimed at pinpointing the demographic, immunological, and viral factors driving these pathological findings, and the role of antiretroviral therapy in reverting these alterations.

Design: B-cell phenotype and correlating factors were evaluated.

Methods: Variations in B-cell subsets were evaluated by flow cytometry in HIV-1infected individuals naive to therapy, elite controllers, and patients treated with antiretroviral drugs (virological control or failure). Multivariable analysis was performed to identify variables independently associated with the B-cell alterations.

Results: Significant differences were observed among patients' groups in relation to all B-cell subsets. Resting memory B cells were preserved in patients naive to therapy and elite controllers, but reduced in treated patients. Individuals naive to therapy and experiencing multidrug failure, as well as elite controllers, had significantly higher levels of activated memory B cells compared to healthy controls. In the multivariate analysis, plasma viral load and nadir $\mathrm{CD}^{+}{ }^{+} \mathrm{T}$ cells independently correlated with major B-cell alterations. Coinfection with hepatitis $C$ but not hepatitis $B$ virus also showed an impact on specific B-cell subsets. Successful protracted antiretroviral treatment led to normalization of all B-cell subsets with exception of resting memory B cells.

Conclusion: Our results indicate that viremia and nadir $\mathrm{CD}^{+}{ }^{+}$cells are important prognostic markers of B-cell perturbations and provide evidence that resting memory B-cell depletion during chronic infection is not reverted upon successful antiretroviral therapy.

(c) 2013 Wolters Kluwer Health | Lippincott Williams \& Wilkins
\end{abstract}

AIDS 2013, 27:1209-1217

Keywords: B cells, CD4 T-cell nadir, coinfection, elite controller, HIV-1, viremia

\footnotetext{
${ }^{a}$ Viral Evolution and Transmission Unit, Division of Immunology, Transplant and Infectious Diseases, ${ }^{b}$ Department of Infectious and Tropical Diseases, San Raffaele Scientific Institute, Milan, Italy, ' Department of Microbiology, Tumor and Cell Biology, Karolinska Institute, dDepartment of Infectious Diseases/Venhälsan, Stockholm South General Hospital, Stockholm, Sweden, Infectious Diseases \& Immunopathology Section, Department of Clinical Sciences, L. Sacco Hospital, University of Milan, and ${ }^{f}$ Human Virology Unit, San Raffaele Scientific Institute, Milan, Italy.

Correspondence to Simone Pensieroso, PhD, Viral Evolution and Transmission Unit, San Raffaele Scientific Institute, Via Olgettina 58, 20132 Milan, Italy.

Tel: +3902 2643 4910; fax: +3902 2643 4905; e-mail: pensieroso.simone@hsr.it

* Francesca Chiodi and Gabriella Scarlatti contributed equally to the writing of the article.

Received: 7 August 2012; revised: 21 December 2012; accepted: 10 January 2013.
} 


\section{Introduction}

During chronic HIV-1 infection, functional perturbations of $\mathrm{B}$ cells take place including hypergammaglobulinemia [1], polyclonal activation [2], and poor immune responses against pathogens and vaccination antigens [3]. These abnormalities may be the consequences of alterations in the physiological development of $\mathrm{B}$ cells, which normally migrate from the bone marrow into the periphery, convert first into transitional and then mature into naive $\mathrm{B}$ cells. Upon antigen encounter, naive B cells differentiate into plasma cells and produce specific antibodies. When infection is cleared, most activated B cells undergo apoptosis, except for some cells constituting the resting memory B-cell pool, which is essential for a rapid secondary immune response.

In the chronic phase of the natural course of HIV-1 infection, the B-cell subsets are altered including resting memory B cells, which are severely depleted and transitional B cells, which are expanded [4]. In addition, aberrant B-cell populations, including activated memory and tissue-like memory (TLM) B cells, which are present at very low levels in HIV-1-negative individuals [5], rise in the blood of HIV-1-infected individuals. These aberrant cells are characterized by the low expression of the surface maker CD21 and display features of immune activation [6] and cellular exhaustion [5]. The factors driving the pathological alterations of B-cell subsets are poorly characterized. Antiretroviral therapy (ART) has only a limited effect on the normalization of the B-cell compartment. Resting memory B cells are maintained if ART is initiated soon after primary infection as shown in both HIV-1-infected children and adults [7]. However, it remains to be elucidated whether ART can ever lead to replenishment of resting memory B cells.

In order to pintpoint the factors involved in pathological changes of $\mathrm{B}$ cells, we investigated whether the different B-cell subpopulations are affected by demographic, immunological, and viral factors in HIV-1-infected individuals. We studied patients with different disease progression, including naive to therapy (NAIVE), elite controllers, and ART-treated patients with and without viral control (ART and ART-FAIL, respectively). Furthermore, to fully understand the impact of ART on the B-cell compartment, we prospectively evaluated the ART-FAIL group after switching to a highly successful therapeutic regimen.

\section{Methods}

\section{Study participants and blood collection}

This is a cross-sectional study including a total of 216 individuals grouped as follows: 144 were HIV-1-infected individuals of whom 47 were NAIVE with plasma viral load above 75 copies $/ \mathrm{ml}, 18$ were elite controllers not eligible for therapy and with at least three plasma HIV-1 RNA determination below 75 copies/ml spanning the 12 months before the blood collection [8], 49 received ART controlling the plasma viral load under detectable levels (ART), and 30 were patients with persistent HIVRNA load more than 75 copies $/ \mathrm{ml}$ despite treatment (ART-FAIL). Seventy-two HIV-1-negative volunteers reporting no diseases and general good healthy conditions were included as healthy controls. Participants' characteristics, which include age, sex, HIV-1 risk factor, hepatitis $\mathrm{B}$ and $\mathrm{C}$ virus ( $\mathrm{HBV}$ and $\mathrm{HCV}$, respectively) coinfection, viral load, $\mathrm{CD} 4^{+}$T-cell counts, and nadir of $\mathrm{CD}^{+} \mathrm{T}$ cells are reported in Table 1. Pairwise comparisons of patients' characteristics are reported in the Table Supplemental digital content 1, http://links. lww.com/QAD/A307. In order to evaluate the relevance of viremia and therapy on causing and ameliorating B-cell dysfunctions, individuals were also stratified in treated (including ART and ART-FAIL individuals) and untreated (including elite controllers and NAIVE) or according to the plasma viral load more than 75 copies $/ \mathrm{ml}$ (including NAIVE and ART-FAIL patients) and less than 75 copies/ml (including elite controllers and ART patients) for multivariable analysis. Furthermore, patients included in the ART-FAIL group were prospectively followed for 144 weeks after switching their failing regimen to a different and successful therapeutic regimen of drugs including raltegravir, etravirine and maraviroc or darunavir [8].

The institution's ethical committees approved the study and all study participants gave a written informed consent. Patient's whole blood was collected at the Stockholm South General Hospital (Sweden), at the San Raffaele Scientific Institute of Milan (Italy), and at the Ospedale Sacco of Milan (Italy). Peripheral blood mononuclear cells (PBMCs) were isolated by Ficoll Gradient centrifugation (Lympholite; Cedarlane, Burlington, North Carolina, USA), frozen, and stored in liquid nitrogen until experiments were performed.

\section{B-cell phenotype}

Multicolor flow cytometry was performed on PBMCs by acquiring $10^{6}$ formaldehyde-fixed cells at the LSRII (Becton Dickinson, Franklin Lakes, New Jersey, USA) or FACS Gallios (Beckman Coulter, Brea, California, USA) flow cytometers. The following mouse antihuman fluorochrome-conjugated monoclonal antibodies (BD) were used: CD19 PERCP-Cy5.5 (clone SJ25C1), CD27 V450 (clone M-T271), CD21 PE (clone B-ly4), and CD10 PE-Cy7 (clone HI101). Dead cells were excluded using the Live/Death Vivid detection kit labeled with a near-infrared dye (Invitrogen, Carlsbad, California, USA). The following gating strategy was used (see Figure, Supplemental Digital Content 2, http://links. lww.com/QAD/A307): only singlets were acquired 


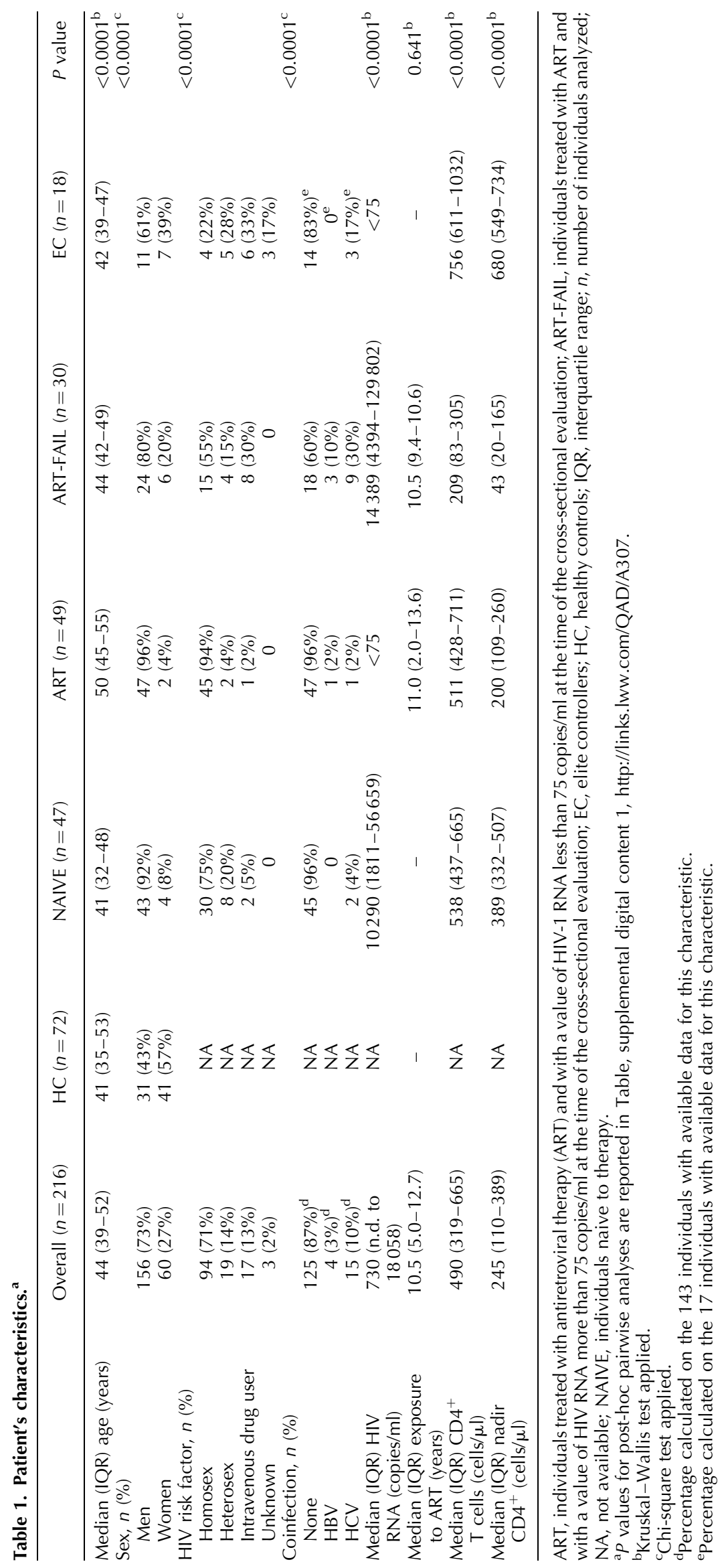

Copyright ( ) Lippincott Williams \& Wilkins. Unauthorized reproduction of this article is prohibited. 
and live B cells were gated on Vivid-CD19 $9^{+}$lymphocytes. Transitional B cells were identified as $\mathrm{CD} 19^{+} \mathrm{CD} 27^{-} \mathrm{CD} 10^{+}$. Thereafter, the $\mathrm{CD}^{-}$cell population was gated and naive $\left(\mathrm{CD} 27^{-} \mathrm{CD} 21^{\text {high+ }}\right)$, resting memory $\left(\mathrm{CD} 27^{+} \mathrm{CD} 21^{\text {high }+}\right)$, activated memory $\left(\mathrm{CD} 27^{+} \mathrm{CD} 21^{-}\right)$, and TLM $\left(\mathrm{CD} 27^{-} \mathrm{CD} 21^{-}\right) \mathrm{B}$ cells were identified. Analyses were performed using FlowJo software version 8.8.3 (Tree Star Inc., Ashland, Oregon, USA).

\section{Statistical analyses}

At univariable analysis, characteristics of the considered participants were reported as median (interquartile range, IQR) or frequency (\%), and were compared by KruskalWallis and Mann-Whitney test or $\chi^{2}$ test. Wilcoxon signrank test was used to assess significant changes from baseline of B-cell subset distributions during the followup study among participants included in the ART-FAIL group. Bonferroni correction was applied for multiple comparisons. Linear relationship between demographic, immunological, and viral parameters with the B-cell subset distributions was assessed by the Spearman rank correlation coefficient.

At multivariable analysis, only HIV-1-infected patients were considered in order to evaluate the influence of HIV-related factors on the distribution of B-cell subsets. A generalized linear model (GLM) was applied to evaluate whether each of the variables (age, sex, HIV-1 risk factor, hepatitis coinfection status, viral load, years of exposure to HIV-1 infection, $\mathrm{CD} 4^{+}{ }^{+}$-cell counts, and nadir $\mathrm{CD} 4^{+} \mathrm{T}$ cells) was independently associated with each B-cell subpopulation. Variables were entered into the multivariable models if their $P$ value was less than 0.05 at univariable analysis, suggesting a different distribution across the patients' groups. Coefficients $(\beta)$ and the corresponding standard errors were estimated and reported; for each categorical variable, the category with the highest sample size was chosen as reference class.

All statistical analyses were performed with SAS software, version 9.2 (SAS Institute, Cary, North Carolina, USA). A two-side $P$ value less than 0.05 was considered statistically significant. Graphs were performed with Prism software v.8 (GraphPad Software Inc., San Diego, California, USA).

\section{Results}

\section{Distribution of B-cell subsets in HIV-1-infected patients and uninfected controls}

In order to verify whether the percentages of B-cell subpopulation were altered among HIV-1-infected individuals and in comparison with healthy controls, the percentages of transitional, naive, resting memory, activated memory, and TLM B cells were evaluated (Fig. 1). Significant differences were observed among groups in relation to all the B-cell subsets (overall comparison: $P<0.0001$ with Kruskal-Wallis test). In addition, significant pairwise comparisons among the (a)

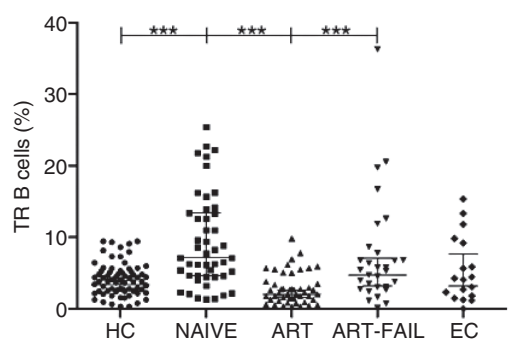

(b)

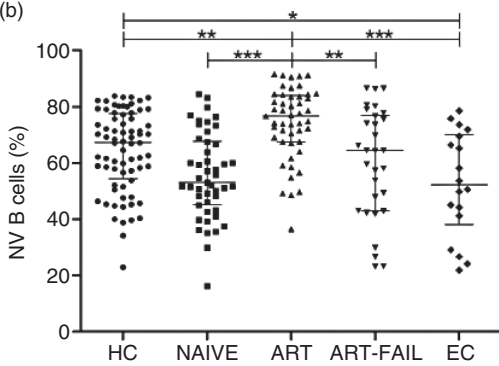

(e)

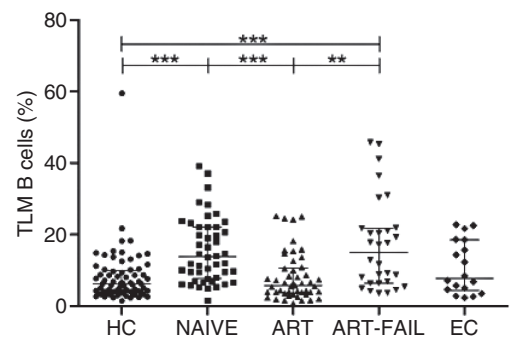

Fig. 1. Phenotype analyses of the B-cell subsets from individual naive to therapy, treated with antiretrovirals (with or without control of viremia) and elite controllers in comparison with healthy individuals (HC). Transitional (a), naive (b), resting memory (c), activated memory (d) and TLM (e) B-cell percentage distributions with median values and interquartile ranges for all the groups of individuals are shown. AM, activated memory; NV, naive; RM, resting memory; TLM, tissue like memory B cells; TR, transitional. $P$ values were evaluated with Mann-Whitney test and Bonferroni correction. $* P<0.05 ; * * P<0.001 ; * * * P<0.0001$. 
groups of HIV-1-infected individuals or in comparison with healthy controls were reported (Fig. 1). Specifically, compared to healthy controls, the transitional B cells were significantly expanded in the NAIVE group, the naive $\mathrm{B}$ cells in the ART, and the activated memory and TLM B cells in NAIVE and ART-FAIL patients. In ART and ART-FAIL individuals, resting memory $\mathrm{B}$ cells were significantly reduced.

B-cell subpopulation distributions in the elite controller group were surprisingly also altered (Fig. 1). They presented with significantly lower percentages of naive and higher percentages of activated memory B cells compared to healthy controls. Elite controllers maintained normal levels of transitional, resting memory, and TLM B cells. Interestingly, they also showed significantly higher levels of resting memory B cells compared to NAIVE as well as ART and ART-FAIL patients.

\section{Role of demographic and HIV-1-related factors in the B-cell subset distribution of HIV-1-infected patients}

In order to investigate factors associated with alteration of B-cell subpopulations, demographic, immunological, and viral characteristics as well as ART exposure were evaluated at multivariable analysis. Viremia was significantly associated with alterations of most B-cell subpopulations (Table 2). Indeed, patients with viral load more than 75 copies $/ \mathrm{ml}$ had higher frequencies of activated memory and TLM and lower naive B cells. The untreated patients were characterized only by higher values of transitional B cells compared to the treated ones. Furthermore, the multivariable analysis evidenced that higher values of resting memory and activated memory $\mathrm{B}$ cells and lower naive B cells were associated with higher values of nadir $\mathrm{CD}^{+} \mathrm{T}$ cells. No alterations were observed in association with absolute $\mathrm{CD} 4^{+} \mathrm{T}$-cell counts. In addition, $\mathrm{HCV}$ coinfection as compared to individuals with no other coinfection, was associated with higher percentages of transitional and naive $\mathrm{B}$ cells, and lower activated memory B cells (Table 2).

Differences in specific B-cell populations were also observed according to HIV-1 risk factor and sex: specifically, homosexual behavior correlated with lower percentages of activated memory; and women showed lower frequencies of resting memory B cells than men. Age did not correlate with any of the B-cell subpopulations.

\section{Control of viral load and the recovery of $\mathrm{CD}^{+} \mathrm{T}$ cells lead to normalization of all B-cell subpopulations except resting memory $B$ cells}

As our results showed a strong association between viral load and the B-cell subset alterations, we evaluated the effects of a protracted and successful antiretroviral regimen on the B-cell compartment in chronic, multidrug-experienced individuals, that is, the ART-FAIL group. After 24 weeks from the start of the new therapeutic regimen, viral load dropped below 75 copies/ $\mathrm{ml}$ in all patients (data not shown). CD4 ${ }^{+}$T-cell counts rose from a median count of 209 (interquartile range: 83.0-305.0) cells $/ \mu \mathrm{l}$ at baseline to 425 (interquartile range: $294.0-609.0 ; P<0.0001)$ cells/ $\mu$ at 24 weeks to finally reach at 144 weeks 497.5 (interquartile range: 333.0-575.0; $P<0.0001$ between baseline and 144 weeks; $P=$ NS between $t=24$ weeks and $t=144$ weeks) cells/ $\mu$ l (data not shown). As shown in Fig. 2, transitional, naive, activated memory, and TLM B-cell distributions returned to normal levels already after 24 weeks of treatment, and the same trend continued thereafter. At 144 weeks, the transitional, activated memory, and TLM were significantly lower than those in healthy controls $(P<0.0001)$ and naive $\mathrm{B}$ cells were significantly higher than those in healthy controls $(P<0.0001)$. In contrast to what noticed with the other subsets, the percentage of resting memory $\mathrm{B}$ cells did not increase and remained below normal levels during the entire follow-up period $(P<0.0001)$. A specific analysis excluded an impact of sex on the resting memory B-cell distribution during ART (data not shown).

In order to further investigate the factors driving the persistence of low percentages of resting memory B cells, we evaluated whether the frequency of resting memory $\mathrm{B}$ cells was linearly associated with viral load and $\mathrm{CD} 4^{+} \mathrm{T}-$ cell counts at all the three time-points of follow-up (i.e., baseline, week 24, and week 144). No correlation was observed between resting memory B cells and viral load at any of the investigated time-points (data not shown). Interestingly, baseline resting memory $\mathrm{B}$ cells were associated with higher $\mathrm{CD} 4^{+} \mathrm{T}$-cell counts at baseline or at 24 weeks $(r=0.54, P=0.002$ and $r=0.51$, $P=0.004$, respectively), but the correlation was lost in relation to the 144 -weeks $\mathrm{CD} 4^{+}$T-cell values $(r=0.23$, $P=0.23$ ). In addition, resting memory $\mathrm{B}$ cells at 24 or 144 weeks did not correlate with $\mathrm{CD}^{+}{ }^{+} \mathrm{T}$-cell counts at any of the investigated time-point (data not shown).

\section{Discussion}

During the natural course of HIV-1 infection, profound alterations of the B-cell compartment occur characterized by hyperactivation of $\mathrm{B}$ cells and consequent hypergammaglobulinemia [1], increase of cell turnover [9], plasmablast differentiation [6], autoantibody production [10], and appearance of cell subpopulations, which are usually not present in uninfected individuals [6]. Introduction of ART was shown to revert most of these alterations $[2,11,12]$, thus suggesting that viremia was the driving force for these impairments. However, whether viremia directly impacts on the B-cell alterations independently of other factors has not been previously evaluated. Here we demonstrate for the first time in a 


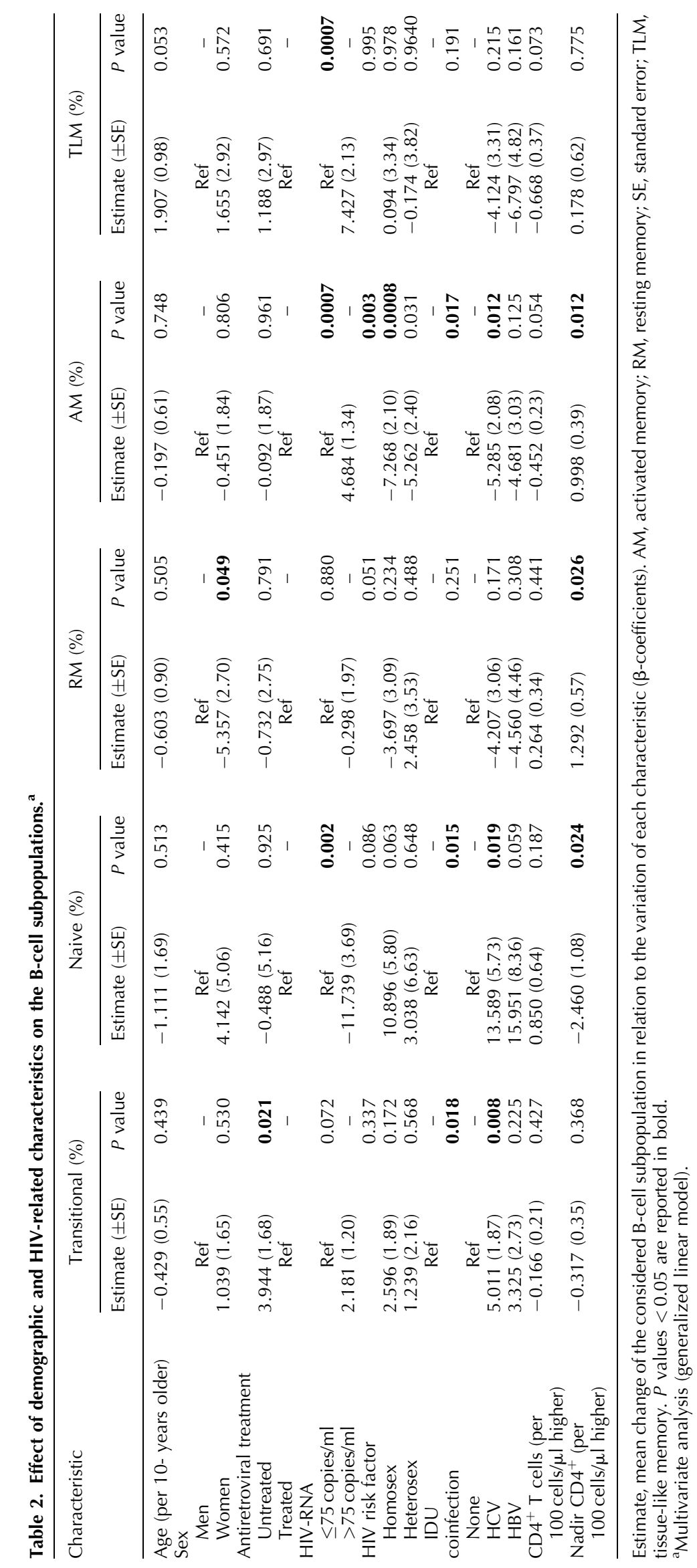


(a)

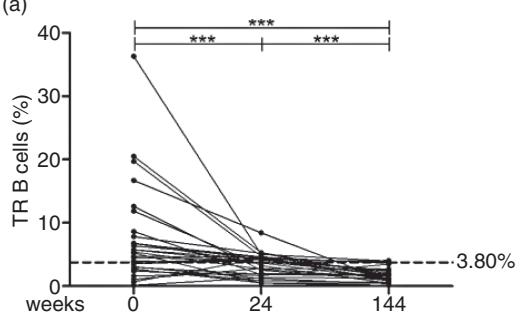

(b)

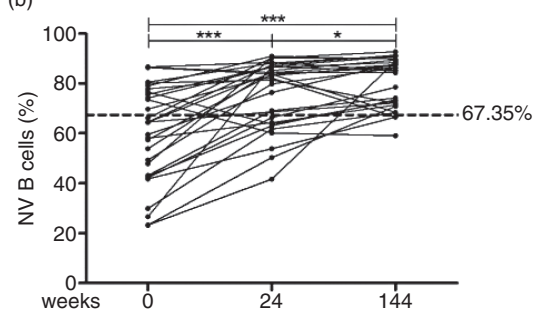

(c)

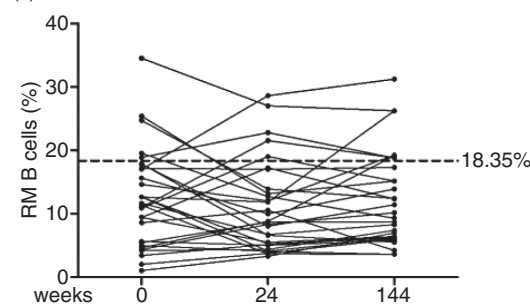

(d)

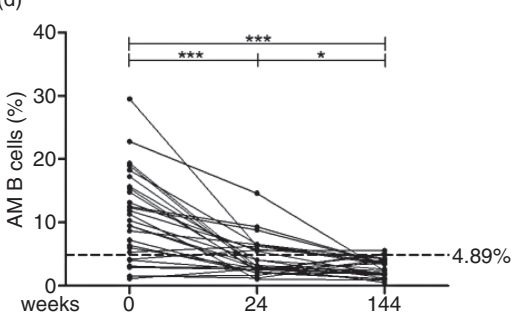

(e)

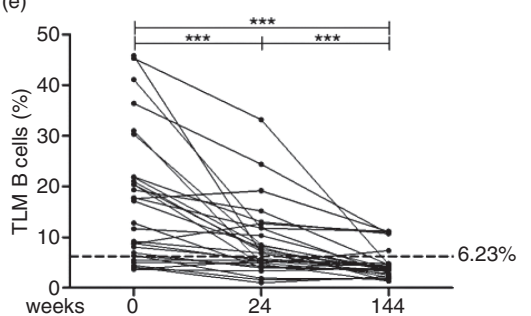

Fig. 2. Trends of the B-cell subsets in ART-FAIL group after administration of a long-lasting successful treatment. Transitional (a), naive (b), resting memory (c), activated memory (d), and TLM (e) B-cell distributions at baseline and after 24 and 144 weeks are reported. Follow-up points of each patient are connected by lines. Dotted lines and reported numbers represent median value percentages of the correspondent subpopulation in the group of HC. $P$ values have been calculated through Wilcoxon sign-rank test. $* P<0.05 * * * P<0.0001$. AM, activated memory; NV, naive; RM, resting memory; TLM, tissue-like memory B cells; TR, transitional.

multivariable analysis that plasma HIV-1 RNA load, independently of other variables, has a direct impact on specific B-cell subpopulations. In fact, in patients with viremia more than 75 copies $/ \mathrm{ml}$, an increase in the percentage of activated memory and TLM B cells and a decrease in the percentage of naive $\mathrm{B}$ cells were shown.

We did not find an association between the $\mathrm{CD} 4^{+}$T-cell count and the frequency of any B-cell population, which is in line with previous data from Malaspina et al. [13]. These authors also observed that, during HIV-1 infection, a decrease in $\mathrm{CD} 4^{+}$T-cell counts was associated with an increase in IL-7 production, which in turn directly correlated with viremia and transitional B-cell increase. In our multivariate analysis, a low number of nadir $\mathrm{CD} 4^{+} \mathrm{T}$ cells showed a strong correlation with low levels of resting memory and activated memory and high levels of naive $\mathrm{B}$ cells. This suggests that loss of $\mathrm{CD} 4^{+} \mathrm{T}$ cells, despite subsequent recovery of the $\mathrm{CD} 4^{+} \mathrm{T}$-cell number, can heavily impact on the physiological maintenance of the B-cell compartment.

Our study demonstrated for the first time that HCV coinfection is an independent factor for B-cell subset alteration, showing a direct correlation with transitional and naive $\mathrm{B}$ cells and an inverse correlation with activated memory B cells. These results indicate that in the same individual, HIV-1 and HCV may synergize to induce similar B-cell alterations. Whether HCV shares analogous mechanisms of action with HIV-1 to affect the B-cell compartment needs still to be investigated. In contrast to our results, Sugalski et al. [14] recently demonstrated that activated memory besides transitional $\mathrm{B}$ cells are increased during chronic HCV infection. This suggests that HCV alone, or in combination with HIV-1, may induce different effects on B-cell subpopulations. Sugalski et al. [14] further showed that serum IL-7 levels or CD4 ${ }^{+}$ T-cell frequency did not correlate with transitional or activated memory B cells percentages during chronic $\mathrm{HCV}$ infection, as instead reported during HIV-1 infection [13], thus suggesting that other factors may be involved in alterations of the B-cell subsets. Interestingly, production of the B-cell activating factor (Blys/BAFF) was shown to correlate with similar alteration of B cells in HCV, HIV-1, and HIV-2 infection [14-17]. Blys/BAFF may, thus, be a common factor driving abnormal activation of $\mathrm{B}$-cell populations during these viral infections.

A goal in the treatment of HIV-1 infection is the recovery of the severely depleted pool of memory B cells taking place during chronic HIV-1 infection [18,19], in order to restore impaired serological memory against HIV-1 and other secondary antigens $[3,20,21]$. Here we show that the percentages of resting memory $\mathrm{B}$ cells from chronically infected patients with multiple failures to ART regimens do not rise despite successful ART for as long as 144 weeks. In previous studies, the increase of memory B cells in chronically HIV-1-infected individuals following ART for various time length was usually slow and incomplete $[4,22,23]$. A recent study by Moir et al. [24], however, showed that resting memory B cells increased significantly after 1 year of ART suggesting that the recovery of resting memory $\mathrm{B}$ cells may be achievable. 
In the study, Moir et al. [24] also demonstrated that patients initiating ART within 6 months from infection showed the higher levels of resting memory B-cells as compared to chronically infected individuals. These data support our previous finding that only early initiation of ART applied within the first phases of infection is associated with preservation of the resting memory B cells in HIV-1-infected children and adults [7,25]. As resting memory B cells are responsible for mounting rapid secondary immune responses, their depletion has an important consequence on the quality of humoral immunity of the patients, with implications for routine vaccination protocols and for the study design of HIV-1 vaccines.

At 144 weeks of therapy, the frequency of the transitional, activated memory, and TLM B-cell subpopulations was significantly reduced in HIV-1-infected patients as compared to healthy controls. This is an interesting finding which deserves further investigation. As the number of naive $\mathrm{B}$ cells also increased significantly in the patients treated with antiretrovirals in relation to healthy controls, the lower frequency of transitional, activated memory, and TLM B cells may only reflect distribution dynamics within the B-cell compartment in the treated patients. As an alternative explanation, it should be considered that activated memory and TLM B cells are activated cells with a pro-apoptotic phenotype [26] and may progressively be depleted over time during antiretroviral therapy.

In this study, we evaluated B-cell subpopulations in a consistent number of elite controllers. Interestingly, resting memory $\mathrm{B}$ cells in this group were maintained at levels comparable to what found in healthy controls, strengthening the relevance of a preserved T-cell compartment to avoid depletion of resting memory $\mathrm{B}$ cells during HIV-1 infection. Surprisingly, the percentages of activated memory B cells in elite controllers were altered, comparable to the increased levels found in NAIVE and ART-FAIL individuals. Whether this is a consequence of low levels of viral replication ongoing at tissue levels or of a generalized immune activation has to be clarified.

\section{Conclusion}

In conclusion, our study clearly showed that HIV-1 viremia and nadir $\mathrm{CD}^{+}{ }^{+} \mathrm{T}$ cells are major and independent determinants together with HCV coinfection in driving the impairment of the B-cell compartment. ART given during chronic HIV-1 infection leads to normalization of all the $\mathrm{B}$-cell subsets with the exception of resting memory B cells. These observations have important clinical consequences as resting memory $\mathrm{B}$ cells are responsible for longlasting memory against pathogens encountered during life and vaccine antigens [27]. Preserving the ability to form and maintain resting memory B cells in HIV-1 individuals by early treatment would ensure a better response to pathogens and vaccines.

\section{Acknowledgements}

The authors would like to thank Nicola Gianotti for fruitful discussions, and Andrea Galli, Bianca Fusetti, and Monica Tolazzi for their technical support. They would like to thank the collaborators from the Units of Infectious Diseases in Northern Italy, particularly Catia Maltempo (SERT ASL 6-7, AOL Magenta, Milan), Giuseppe Paraninfo and Giampiero Carosi (Department of Infectious and Tropical Medicine, University of Brescia, Brescia), Gianpaolo Quinzan and Fredy Suter (Division of Infectious Diseases, Ospedali Riuniti, Bergamo), Ercole Concia, (Unit of Infectious Diseases, Ospedale Policlinico GB Rossi, Verona), Rosangela Beretta, Michela Fasolo, Paola Meraviglia, Carlo Magni, Amedeo Capetti and Giuliano Rizzardini (I and II Unit of Infectious Diseases, L. Sacco Hospital, Milan), Francesco Mazzotta and Massimo Di Pietro (Department of Infectious Diseases, S.M. Annunziata Hospital, Florence), Elisabetta Blasi Vacca, Antonio Di Biagio and Claudio Viscoli (Department of Infectious Diseases, San Martino Hospital, University of Genoa), Maria Pia Allegri (U. O. di Malattie Infettive, Ospedale della Misericordia, Grosseto); Paula Castelli (Unità Operativa di Malattie Infettive, Macerata).

S.P., G.S., and F.C. contributed to the study's conception and design, and wrote the paper.

S.N., A.C., G.T., B.H., A.R., and M.M. contributed to the collection of specimens from patients, clinical data collection and elaboration, and followed the patients during the study.

L.G. performed statistical analyses, contributed to the interpretation of the results and in the writing of the article.

S.P., N.R., and D.M. performed experiments.

All authors contributed to the completion of the paper.

Financial support was received from the European Commission funded projects EUROPRISE-Network of Excellence grant number LSHP CT-2006-037611 and NGIN grant number 201433. Further financial support was received by the ELVIS cohort study (Grant ELVIS 10G68 from Istituto Superiore di Sanità), and from the Swedish International Development Agency and the Swedish Medical Research Council.

\section{Conflicts of interest}

All the authors declare to have no competing financial interests. 


\section{References}

1. Lane HC, Masur H, Edgar LC, Whalen G, Rook AH, Fauci AS. Abnormalities of B-cell activation and immunoregulation in patients with the acquired immunodeficiency syndrome. N Engl J Med 1983; 309:453-458.

2. Morris L, Binley JM, Clas BA, Bonhoeffer S, Astill TP, Kost R, et al. HIV-1 antigen-specific and -nonspecific $B$ cell responses are sensitive to combination antiretroviral therapy. I Exp Med 1998; 188:233-245.

3. Hart M, Steel A, Clark SA, Moyle G, Nelson M, Henderson DC, et al. Loss of discrete memory $B$ cell subsets is associated with impaired immunization responses in HIV-1 infection and may be a risk factor for invasive pneumococcal disease. I Immunol 2007; 178:8212-8220.

4. De Milito A, Morch C, Sonnerborg A, Chiodi F. Loss of memory (CD27) B lymphocytes in HIV-1 infection. AIDS 2001; 15:957964.

5. Moir S, Ho J, Malaspina A, Wang W, DiPoto AC, O'Shea MA, et al. Evidence for HIV-associated B cell exhaustion in a dysfunctional memory B cell compartment in HIVinfected viremic individuals. J Exp Med 2008; 205:17971805.

6. Moir S, Malaspina A, Ogwaro KM, Donoghue ET, Hallahan CW, Ehler LA, et al. HIV-1 induces phenotypic and functional perturbations of $B$ cells in chronically infected individuals. Proc Natl Acad Sci U S A 2001; 98:10362-10367.

7. Pensieroso S, Cagigi A, Palma P, Nilsson A, Capponi C, Freda E, et al. Timing of HAART defines the integrity of memory B cells and the longevity of humoral responses in HIV-1 verticallyinfected children. Proc Natl Acad Sci U S A 2009; 106:79397944.

8. Nozza S, Galli L, Bigoloni A, Nicola G, Pogliaghi M, Cossarini F, et al. Durability and safety of a novel salvage therapy in R5-tropic HIV-infected patients: maraviroc, raltegravir, etravirine. J Acquir Immune Defic Syndr 2011; 56:e113e115.

9. Moir S, Malaspina A, Pickeral OK, Donoghue ET, Vasquez J, Miller NJ, et al. Decreased survival of $\mathbf{B}$ cells of HIV-viremic patients mediated by altered expression of receptors of the TNF superfamily. I Exp Med 2004; 200:587599.

10. Shirai A, Cosentino M, Leitman-Klinman SF, Klinman DM. Human immunodeficiency virus infection induces both polyclonal and virus-specific B cell activation. I Clin Invest 1992; 89:561-566.

11. Notermans DW, de Jong JJ, Goudsmit J, Bakker M, Roos MT, Nijholt $L$, et al. Potent antiretroviral therapy initiates normalization of hypergammaglobulinemia and a decline in HIV type 1-specific antibody responses. AIDS Res Hum Retroviruses 2001; 17:1003-1008.

12. Jacobson MA, Khayam-Bashi $\mathrm{H}$, Martin JN, Black D, Ng V. Effect of long-term highly active antiretroviral therapy in restoring HIV-induced abnormal B-lymphocyte function. J Acquir Immune Defic Syndr 2002; 31:472-477.
13. Malaspina A, Moir S, Ho J, Wang W, Howell ML, O'Shea MA, et al. Appearance of immature/transitional B cells in HIV-infected individuals with advanced disease: correlation with increased IL-7. Proc Natl Acad Sci U S A 2006; 103:2262-2267.

14. Sugalski JM, Rodriguez B, Moir S, Anthony DD. Peripheral blood B cell subset skewing is associated with altered cell cycling and intrinsic resistance to apoptosis and reflects a state of immune activation in chronic hepatitis $\mathrm{C}$ virus infection. J Immunol 2010; 185:3019-3027.

15. Tarantino G, Marco VD, Petta S, Almasio PL, Barbaria F, Licata $A$, et al. Serum BLyS/BAFF predicts the outcome of acute hepatitis C virus infection. I Viral Hepat 2009; 16:397-405.

16. Sene D, Limal N, Ghillani-Dalbin P, Saadoun D, Piette JC, Cacoub P. Hepatitis C virus-associated B-cell proliferation: the role of serum B lymphocyte stimulator (BLyS/BAFF). Rheumatology (Oxford) 2007; 46:65-69.

17. Tendeiro R, Fernandes S, Foxall RB, Marcelino JM, Taveira N, Soares RS, et al. Memory B-cell depletion is a feature of HIV-2 infection even in the absence of detectable viremia. AIDS 2012; 26:1607-1617.

18. De Milito A. B lymphocyte dysfunctions in HIV infection. Curr HIV Res 2004; 2:11-21.

19. Cagigi A, Nilsson A, De Milito A, Chiodi F. B cell immunopathology during HIV-1 infection: lessons to learn for HIV-1 vaccine design. Vaccine 2008; 26:3016-3025.

20. De Milito A, Nilsson A, Titanji K, Thorstensson R, Reizenstein E, Narita $M$, et al. Mechanisms of hypergammaglobulinemia and impaired antigen-specific humoral immunity in HIV-1 infection. Blood 2004; 103:2180-2186.

21. Titanji K, De Milito A, Cagigi A, Thorstensson R, Grutzmeier S, Atlas A, et al. Loss of memory B cells impairs maintenance of long-term serologic memory during HIV-1 infection. Blood 2006; 108:1580-1587.

22. Moir S, Malaspina A, Ho J, Wang W, Dipoto AC, O'Shea MA, et al. Normalization of $B$ cell counts and subpopulations after antiretroviral therapy in chronic HIV disease. I Infect Dis 2008; 15:572-579.

23. D'Orsogna LJ, Krueger RG, McKinnon EJ, French MA. Circulating memory B-cell subpopulations are affected differently by HIV infection and antiretroviral therapy. AIDS 2007; 21:17471752.

24. Moir S, Buckner CM, Ho J, Wang W, Chen J, Waldner AJ, et al. B cells in early and chronic HIV infection: evidence for preservation of immune function associated with early initiation of antiretroviral therapy. Blood 2010; 116:5571-5579.

25. Titanji K, Chiodi F, Bellocco R, Schepis D, Osorio L, Tassandin C, et al. Primary HIV-1 infection sets the stage for important B lymphocyte dysfunctions. AIDS 2005; 19:1947-1955.

26. Rethi B, Sammicheli S, Amu S, Pensieroso S, Hejdeman B, Schepis D, et al. Concerted effect of lymphopenia, viremia and $T$ cell activation on Fas expression of peripheral $B$ cells in HIV-1 infected patients. AIDS [Epub ahead of print].

27. Yoshida T, Mei H, Dorner T, Hiepe F, Radbruch A, Fillatreau S, Hoyer BF. Memory B and memory plasma cells. Immunol Rev 2010; 237:117-139. 